\title{
The Analysis of Stiffness for Rubbery Metallic Material Based on Mesoscopic Features
}

\author{
Hong Zuo ${ }^{1 *}$, Hongbai Bai ${ }^{2}$, Yuhong Feng ${ }^{3}$ \\ ${ }^{1}$ MOE Key Laboratory for Strength and Vibration, School of Aerospace, Xi'an Jiaotong University, Xi'an, China; ${ }^{2}$ Department of \\ Gun Engineering, Ordnance Engineering College, Shijiazhuang, China; ${ }^{3}$ School of Material Science \& Engineering, Xi'an Jiaotong \\ University, Xi'an, China. \\ Email: zuohong@mail.xjtu.edu.cn
}

Received November $23^{\text {rd }}, 2010$; revised December $15^{\text {th }}, 2010$; accepted May $17^{\text {th }}, 2011$.

\begin{abstract}
In this article, deformation and mechanical response of a rubbery metallic material were investigated. First, the mesoscopic structural properties of the material and its evolution during part producing were analyzed and described in detail. Then the inherence relationship between the macroscopic mechanical properties and mesoscopic structural characteristics were studied, in which the related mesoscopic structural characteristics were limited in the basic unit ( $\mathrm{mm})$ scale such as the radius of metal wire and unit coil, etc. Furthermore, according to the mesoscopic properties of the material, a curved beam unit based on the mesoscopic scale and shape factor was introduced to bridge the mechanical response and the mesoscopic parameters such as the beam orientation and spatial distribution. In the end, a mesoscopic stiffness model was proposed, from which the macroscopic mechanical properties of material could be deduced from the mesoscopic characteristic size, shape and the mechanical properties of base metallic material.
\end{abstract}

Keywords: Rubbery Metallic Material, Stiffness Model, Mesoscopic Characteristic

\section{Introduction}

In recent years, a kind of rubbery metallic material (RMM) has been applied as some kinds of seal material, heat shield material, filters, gaskets and aircraft engine mounts in a large number of industrial fields where crude rubber material have been usually served, owing to its excellent applicability of environment and longer life. Among these applications, the most exciting lies in its application in aerospace as a vibration absorber since the space environment needs the absorber possesses a wide range of temperature adaptability and more than several years life, while crude rubber cannot competent for. Except of this, this material also has lower density and excellent mechanical properties. Considering the mechanical behavior such as the stiffness and deformation responding for the applied loads play an important role during the damping material design and application, it should be further investigated.

Although RMM has been applied as a damping part in the aerospace for a longtime, there still need sufficient investigation in several fields such as its application and mechanical behavior. According to the studies from open literature, we only find that fewer tests conducted by
Childs [1], which relate to this material for possible application in the high-pressure fuel turbo pump on space shuttle. The results of their bench test showed good damping characteristics, but numerical quantities were not reported. A computer rotor dynamic model, with RMM damper installed in the supports was reported to have much better performance than any other alternative available. The use of RMM as vibration isolators was described by Rivin [2] and Barnes [3], they suggested the density of the RMM influences the stiffness to great extent when used RMM for aircraft engine mounts. They also noted the damping characteristics of RMM are dependent on the material selected. As a bearing damper in a liquid hydrogen turbo pump, RMM was applied in the LE-7 engine for the first time reported by Okayasu and others [4]. The old turbo pump with no RMM damper faced several vibration problems. Whereas, once RMM "friction dampers" applied in the bearing supports of the turbo pump, the machine can work in a high rate, larger than its third critical speed. They reported that RMM show a most effective source of damping compared with other damping units. The experiment results from Wang and $\mathrm{Zhu}$ [5] show RMM damper could control three times imbalance than the squeeze film damper. Vance 
and co-workers [6-8] reported that RMM dampers in parallel with a squirrel cage allow wider control of the support stiffness. They showed RMM could work even at cryogenic temperatures. They also conducted the endurance tests on these dampers over six months and showed little to no change in the damping characteristics. They reports the dynamic characteristics of the material are dependent on the radial strain amplitude and axial strain (compression), and they also suggested the effect of axial thickness of the mesh have to be studied.

It is well-known that material mechanical response is usually influenced by many factors, e.g., the parameters from forming procedures, mechanical properties and size of the base mater. However, there are few investigations in this field were obtained from the open literatures till now. Especially, there has no study focused on the mesoscopic characteristic and stiffness response was proposed. Thus, the purpose of the present study is to investigate the relationship between the mesoscopic features of the material and macroscopic stiffness behavior during the material subjected to a compressed loading along the direction of its stamping axis during forming.

In this paper, through investigating the evolution rule of several mesoscopic feature parameters such as shape, size and spatial distribution of base mater coil before and after stamping during part producing, a mesoscopic stiffness model which could represent the mesoscopic properties and macroscopic mechanical properties of the material was proposed based on the mechanical analysis.

\section{Mesoscopic Characteristics of RMM}

Before discussing mesoscopic characteristics of RMM, the detailed produced procedures should be expatiated firstly as follows: first, the metal wire with its base material as stainless steel, the refractory alloy or low temperature alloy was wrapped to spring line. Then, the spring line was knitted to body as roughcast of RMM with different style, e.g., the three-dimension knitted method or the wool clew circled method. Third, the roughcast was molded to the designed part through cold stamping. Whereas, it is the excellent environmental adaptability of the base metal endow RMM with good temperature adaptability and longer life same as base material. On the other hand, this forming procedure for roughcast could be also thought as three steps of three-dimensional molding, i.e., the first step is to create a "line" as the filamentous spring with equal pitch. The next step is to knit a "plane" through a crossed or slanted knitting technique by spring "line", similarly to the blanket knitting (Figure 1). The last step is to create "body" through different techniques such as the "plane" piling up.

Expect for the physical properties such as the me- chanical properties, the friction coefficient, and the extent of temperature adaptability of the base material, the mesoscopic characteristic parameters of RMM usually contains the geometric size of the mesoscopic structure and the characteristics of base material, for example, the diameter of the metallic wire $d_{s}$, the diameter of spirals $d_{j}$, the nominal included angle of the definite metallic wire with the plane of the compressed surface $\alpha$, and the relative density of RMM $\bar{\rho}$.

According to the procedures of weave and cold stamped extent, the relationship between the relative density of RMM and mesoscopic structure feature parameter (the diameter of metallic wire, the diameter of spirals and the compressed extent) could be derived according to several proper assumptions: the homogeneous assumption, the self-similar deformation assumption in the mesoscopic view and the well-proportional deformation assumption during cold stamping stage. Examining the mesoscopic structure features of the material before cold stamping, it will be found that the typical mesoscopic structure appears as an element cubic composed with two crossed circles of metallic wire. This can be thought as a self-similar elementary material unit for RMM, as shown in Figure 2. According to the definition of relative density $\bar{\rho}$ for porous material the ratio of the volume of related base material with the porous material, the relative density of a RMM is expressed as follows according to the spatial arrangement of metallic wire (Figure 3):

$$
\bar{\rho}=\frac{\pi \sqrt{\pi^{2}+1} d_{j} d_{s}^{2}}{2 \mu\left(d_{j}+d_{s}\right)^{3}}
$$

where $d_{j}$ denotes the pitch diameter of the spiral, $d_{s}$ the diameter of the metallic wire, and $\alpha$ the included

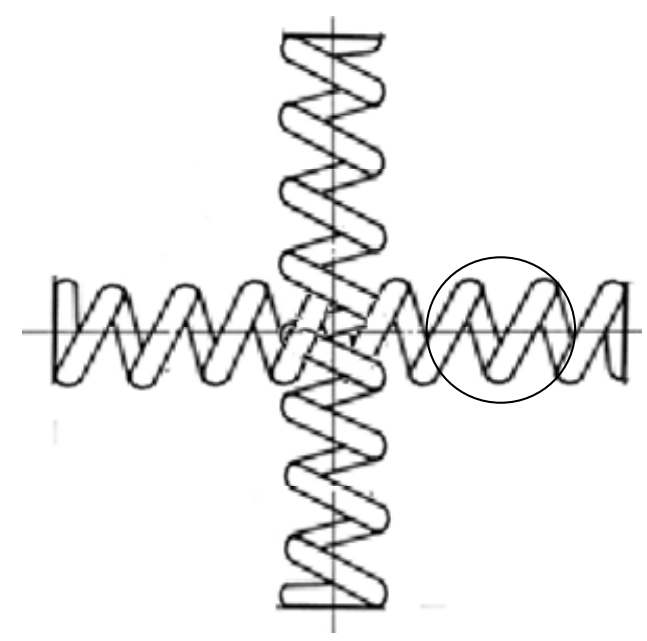

Figure 1. The crossed weave or oblique weaves techniques by the "line". 


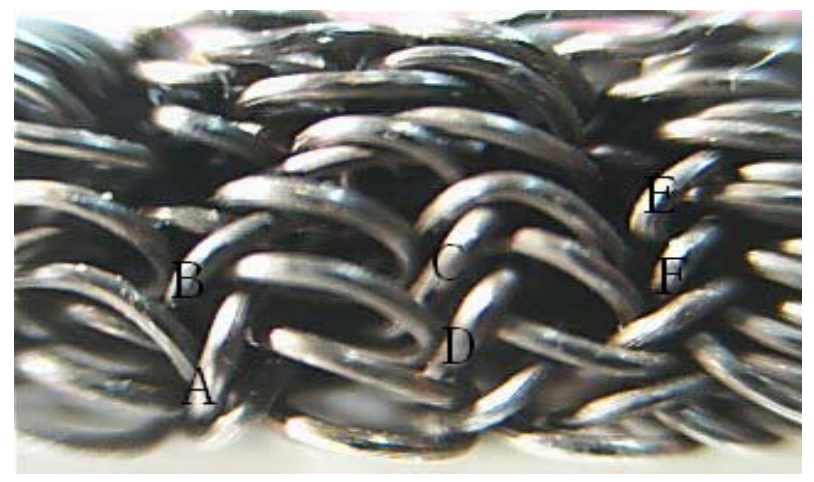

Figure 2. The mesoscopic feature of metallic wire on the side surface.

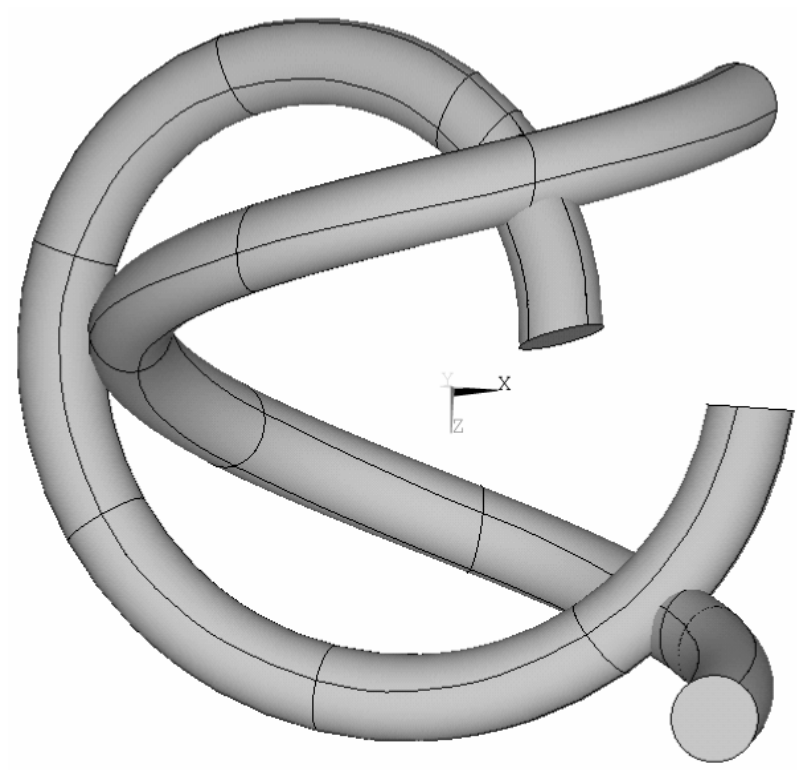

Figure 3. The spatial configuration of metallic wire.

angle of metallic wire. For the original roughcast before cold stamping, $\mu$ is the compressed ratio during cold stamping and can be related to the included angle of metallic wire $\alpha$ in one circle wires, $\tan \alpha=\mu / \pi . \mu=1$ corresponds to the no cold stamping body while $\mu<1$ corresponds to the stage of cold stamping. For example, for the case of no cold stamping, $\alpha=\arctan (1 / \pi)$ (about $17.66^{\circ}$ ) based on the assumption that two circle wires crossed in a cubic body, and the relative density $\bar{\rho}_{0}$ can be expressed as follows:

$$
\bar{\rho}_{0}=\frac{\pi \sqrt{\pi^{2}+1} d_{j} d_{s}^{2}}{2\left(d_{j}+d_{s}\right)^{3}}
$$

During cold stamping, compression deformation along the axis of cold stamping happened. And on the directions perpendicular to this axis, no deformation take placed from the macroscopic view since the rigid con- straint of material by the die. Therefore, the mesoscopic deformation and shape change of metallic wire during the macroscopic uniaxial compressed deformation can be described as follows. First, the included angle between the metallic wire and compressed plane $\alpha$ decreased gradually with the macroscopic deformation increased. Next, the metallic wire deformed in a compound of twisting and bending, as shown in Figure 4. It is obvious that the influence of this complex deformation on the relative density of material is smaller than the decrease of included angle. Thus, it is reasonable to evaluate the change of relative density only through the change of included angle.

Suppose $d_{j}$ and $d_{s}$ were given, the relative density $\bar{\rho}_{0}$ of material was determined by Equation (2). For example, if $d_{j}$ is taken as $1.0 \mathrm{~mm}$, and $d_{s} 0.1 \mathrm{~mm}$ before cold stamping, then $\bar{\rho}_{0}$ is determined as 0.128 . In general, it is difficult to calculate the relative density through Equation (1) since the compressed ratio is not easily determined after cold stamping. However, the relative density of the material before cold stamping can be obtained by experimental measurement. Therefore, we can determine the included angle corresponding to the material after cold stamping through relative density. Based on the definition of included angle of wire in the material unit and the homogeneous assumption, the self-similar assumption and the proportional deformation assumption above (referred to Figure 5), the nominal included angle of wire for material is defined by means of the relation of included angle of wire with other parameters in Equation (1),

$$
\alpha=\arctan \frac{\sqrt{\pi^{2}+1} d_{j} d_{s}^{2}}{2 \bar{\rho}\left(d_{j}+d_{s}\right)^{3}}
$$

For example, with the same values of $d_{j}$ and $d_{s}$, if $\bar{\rho}$ is increased to 0.2 , then, $\alpha$ will decrease to $3.54^{\circ}$. In this case, the volume ratio $\mu$ of compressed model and original model is nearly 0.19 from the geometrical relation of $\alpha$ and $d_{j}$.

\section{The Stiffness Model}

Once the included angle of two crossed wires in a unit was given, the spatial distribution of wires in a unit can be determined. Given the mechanical properties of metallic wire, the stiffness of this unit is derived based on the deformation stiffness analysis (referred to Figure 5).

For the material RMM, it is reasonable to select two crossed wire circles as a representative unit to represent the material mesoscopic structure (referred to Figure 3). In this material element, there exist eight same deformed metallic wire segments distributed in the space of the element and stacking each other. These segments have a 


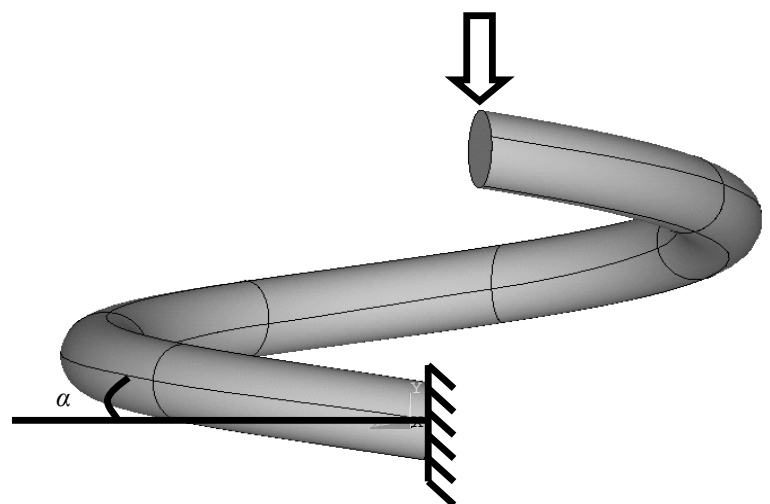

(a)

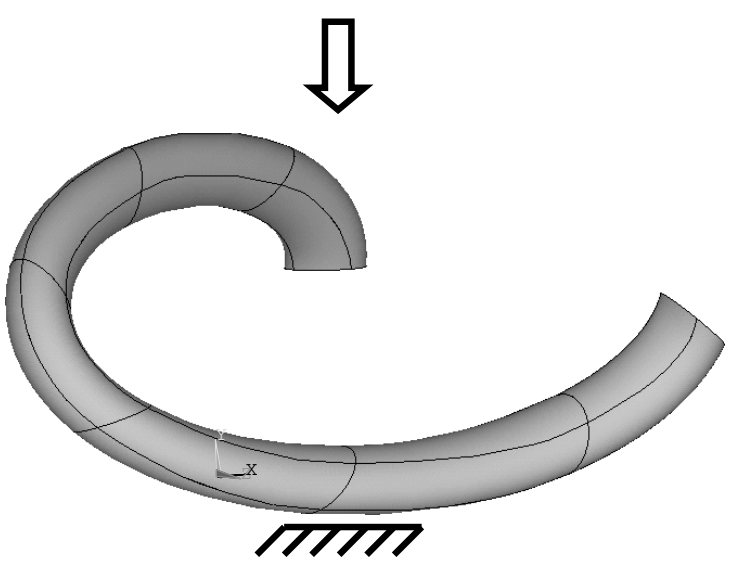

(b)

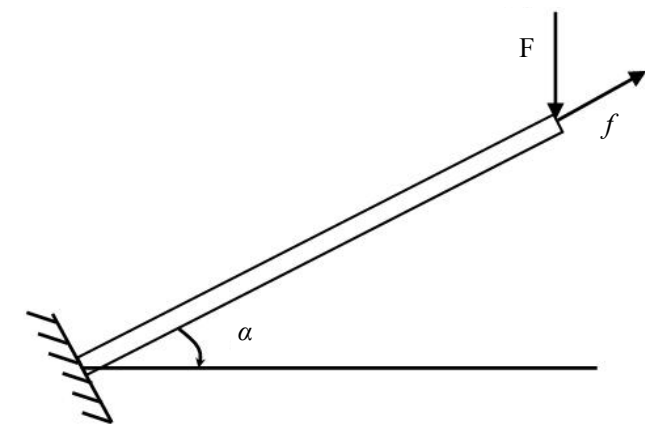

(c)

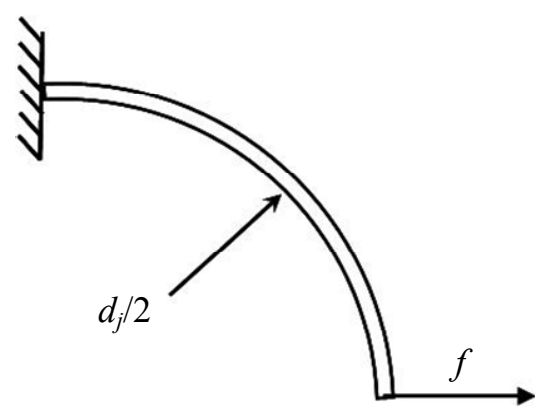

(d)

Figure 4. The small curve beam model. length of quarter of the wire circle and can be thought as a basic element comprising the representative unit of the material according the space distribution (referred to Figure 3). Thus, the stiffness of the unit is thought as the basic element stacked in series by four layers and in each layer there are two basic elements arranged parallel. Now let's check the deformation of this basic unit when the compressed loading subjected, we can find that this basic unit can be thought as part of a curve cantilever beam illustrated in Figure 4. The constraint of curved cantilever beam is as follows: the fixed constraint with six freedoms at one end, and two forces stressed on the other end of the cantilever, one is the pressure force along the orientation of stamping direction, and the other is the constraint force by the die around. In practice, this constraint force in the units witch don't contact with the die can be though as the frictional force owing to the relative sliding in the surface of adjacent metallic wires (shown in Figure 4). Thus, according to the basic deformation analysis, the stiffness of the curve beam is derived as follows:

$$
k_{e}=\frac{d_{s}^{4}}{16 d_{j}^{3}\left(d_{j}+d_{s}\right)}\left(\frac{\cos ^{2} \alpha}{2 G}\left(\frac{3}{4}-\frac{2}{\pi}\right)+\frac{2-f \sin 2 \alpha}{8 E}\right)^{-1}
$$

Where $G$ and $E$ are the shear module and Young's module of the metallic wire respectively, $\alpha$ is the included angle calculated by relative density of RMM, and $f$ is the friction coefficient of base material.

A representative unit in the material is composed by 8 basic elements (curved beams) with same deformation manner. According to the contribution of this element to the representative unit stiffness, the stiffness of one representative unit is calculated here:

$$
K=\frac{d_{s}^{4}}{32 d_{j}^{3}\left(d_{j}+d_{s}\right)}\left(\frac{\cos ^{2} \alpha}{2 G}\left(\frac{3}{4}-\frac{2}{\pi}\right)+\frac{2-f \sin 2 \alpha}{8 E}\right)^{-1}
$$

where $\alpha=\arctan \frac{\sqrt{\pi^{2}+1} d_{j} d_{s}^{2}}{2 \bar{\rho}\left(d_{j}+d_{s}\right)^{3}}$. From the expression of the stiffness of the unit, as soon as $d_{j}$ and $d_{s}$ were given, for a metal wire with a definite $E, G$ and $f$, the stiffness of metallic rubber is then a function of $\bar{\rho}$.

\section{Discussion}

According to the mesoscopic stiffness model proposed above, the effect of each mesoscopic characteristic parameter such as $d_{j}, d_{s}$ and $\bar{\rho}$ on the macroscopic material stiffness could be further discussed.

\subsection{The Influence of $d_{j}$ and $d_{s}$}

The pitch diameter $d_{j}$ of spiral of the wire is a main 

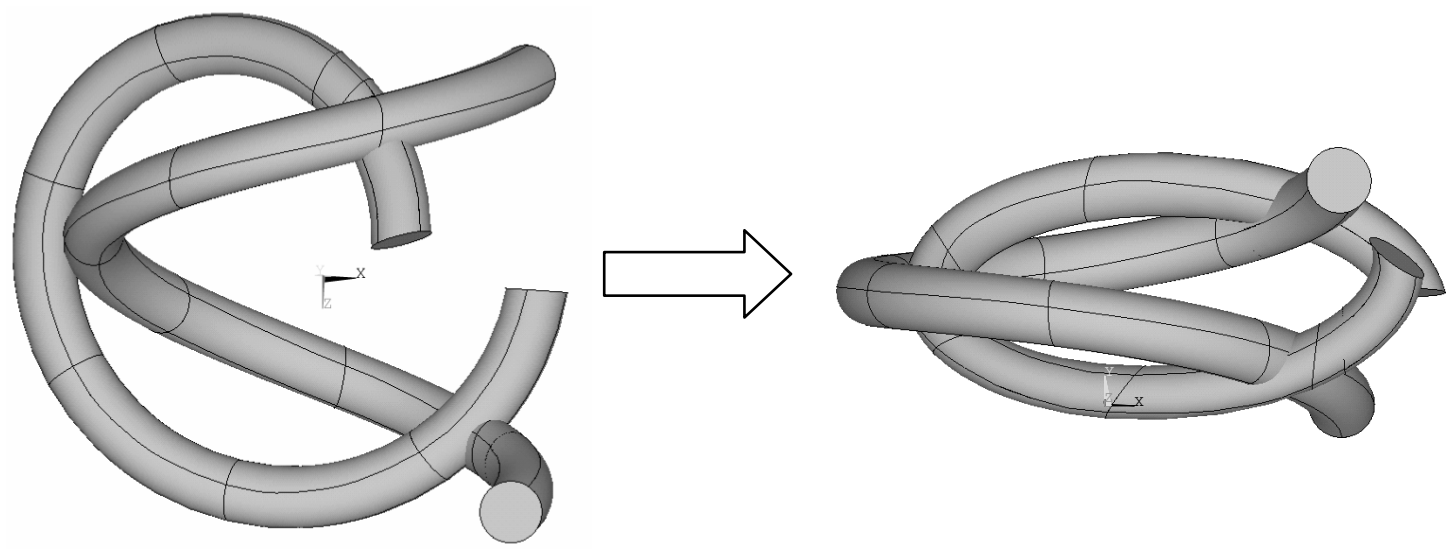

Figure 5. The mesoscopic deformation process in RMM.

control parameter during material production. Although it is well-known that it has a considerable effect on the total stiffness of material, however this effect and its variation were never exactly evaluated analytically in the past. In general, the diameter of metallic wire $d_{s}$ is about tenth of pitch diameter of spiral, thus the value of $\left(d_{j}+d_{s}\right)$ usually is estimated as $d_{j}$ approximately when evaluating the influence of $d_{j}$. Therefore, through the expression of Equation (5), the magnitude of stiffness of the material is determined in inverse proportion to the fourth power of $d_{j}$ approximately. The effect of the pitch diameter of spiral $d_{j}$ on the stiffness according to the relative experiments [9] was illustrated in Figure 6. In the figure, the experimental result expressed as a solid curve while the predicted result was denoted as a dashed curve. From the figure, the variation of the stiffness vs. increase of $d_{j}$ is consistent with the prediction by Equation (5).

The diameter of metallic wire $d_{s}$ is another mesoscopic material feature which influences the mechanical properties of the material and is determined during the material design. From Equation (5), the stiffness of the material RMM is in a direct proportion to the fourth power of $d_{s}$, and this can be compared with the experimental result illustrated in Figure 7. As seem as in the Figure 6, the experimental result of stiffness expressed as a solid curve while the predicted result was denoted as a dashed curve. From the figure, the increase trend of stiffness with $d_{s}$ is measure also the direct proportion to the fourth power of $d_{s}$, the same as the predicted by the proposed model.

If we introduce another parameter $d_{r}$ to denote the ratio of the diameter of spiral $d_{j}$ and the diameter of wire $d_{s}$, then, Equation (5) is expressed as follows

$$
K=\frac{N}{32 d_{r}^{3}\left(d_{r}+1\right)} \text {. }
$$

where

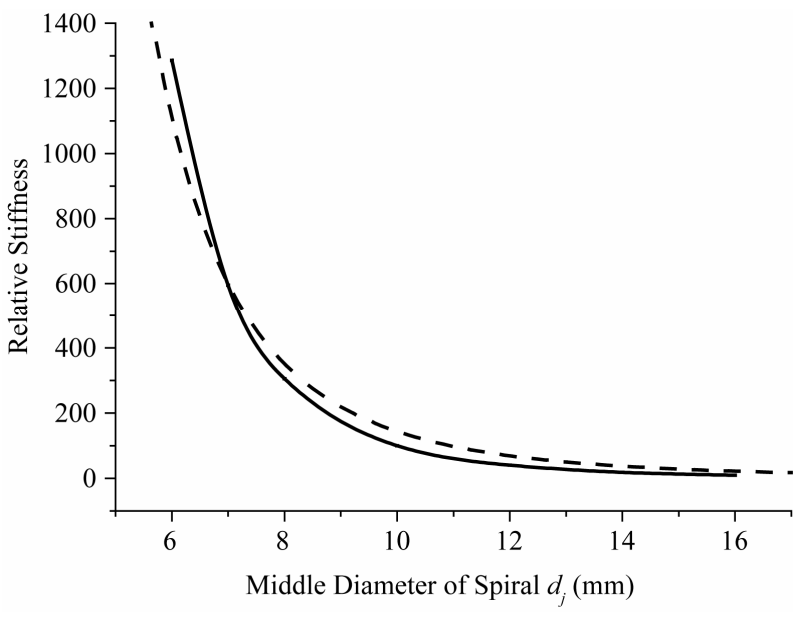

Figure 6. The influence of the diameter of the spiral of wire.

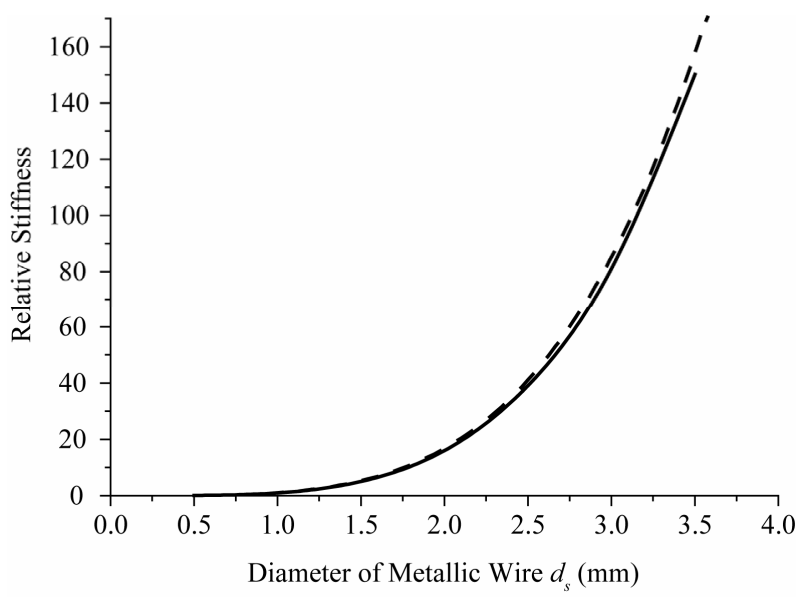

Figure 7. The influence of the diameter of metallic wire.

$$
N=\left(\frac{\cos ^{2} \alpha}{2 G}\left(\frac{3}{4}-\frac{2}{\pi}\right)+\frac{2-f \sin 2 \alpha}{8 E}\right)^{-1}
$$


Here, $N$ expresses the mechanical properties of material and the intensity of cold stamping, while $d_{r}$ expresses the mesoscopic size properties of the original roughcast material. It is obviously from Equation (6) that a single parameter, the ratio of diameter of spiral and wire $d_{r}$ can represent the influence of mesoscopic properties quantitively.

\subsection{The Influence of $\bar{\rho}$}

Remember that there exist an inherent relation between the macroscopic feature $\bar{\rho}$ and the mesoscopic parameter $\alpha$, referenced to Equation (3) owing to the cold stamped processing of the material. According to the production procedure of RMM, the magnitude of relative density $\bar{\rho}$ of the material is controlled by the intensity of compressed deformation along the direction of compressed force, and has nothing to do with the deformation along other directions. Now, let's consider the other expression of Equation (5) as follows

$$
K=\frac{M}{\cos ^{2} \alpha}\left(\frac{1}{2 G}\left(\frac{3}{4}-\frac{2}{\pi}\right)+\frac{1}{4 E}-\frac{f L}{4 E \bar{\rho}}\right)^{-1},
$$

where $M=\frac{1}{32 d_{r}^{3}\left(d_{r}+1\right)}$ and considering $\tan \alpha \propto 1 / \bar{\rho}$.

Here, $\mathrm{L}$ is a coefficient and can be determined by Equation (3). In practice, the included angle $\alpha$ is very small $\left(<5^{\circ}\right)$, thus $\cos \alpha$ is equal to 1 approximately, thus, the flexibility of RMM $(1 / \mathrm{K})$ is decreased inverse proportionally with the relative density $\bar{\rho}$ increased. However, on the other hand, if the included angle is very small, the intervention between contacted wires is intensively. Thus assumption of freedom deformation for curve cantilever is no longer active, and this influence should be investigated further.

\subsection{The Influence of Friction Coefficient of Metallic Wires $f$}

During the material deformed under compressed loading, its mesoscopic deformation experienced several stages. After the early stage where no relative slide of contacted wires, the influence of friction coefficient of metallic wires $f$ on the stiffness of the material emerged in the deformation later. It is obviously from Equation (5) that the friction coefficient of metallic wires will affect the value of the stiffness of material. The relationship of the friction coefficient of metallic wires $f$ and the total stiffness of material shows the larger friction coefficient will induce the higher stiffness of the material. The flexibility of RMM is decreased proportionally with the friction coefficient of metallic wires $f$ increased.

\section{Conclusions}

From the mesoscopic stiffness model proposed in this study, the influence of mesoscopic structure features and material properties on the macroscopic stiffness of the material have been evaluated quantitatively. According to this model, it is possible to design the macroscopic property of the material at early producing stage. The main conclusions of this study are presented below:

1) The variation law of stiffness of the material RMM is in inverse proportional to the fourth power of $d_{j}$ approximately.

2) The stiffness of the material RMM is in direct proportion to the fourth power of $d_{s}$, although the value of $d_{s}$ is smaller than the value of $d_{j}$.

3) The larger relative density of material and the larger friction coefficient of metallic wires will strengthen the macroscopic stiffness of the material.

\section{Acknowledgements}

This work was supported by the National Defense Foundation, the Natural Science Foundation of Xi' an Jiaotong University and the Natural Science Foundation of Shanxi (2005A19).

\section{REFERENCES}

[1] D. W. Childs, "The Space Shuttle Main Engine HighPressure Fuel Turbopump Rotordynamic Instability Problem," ASME Journal of Engineering for Power, Vol. 100, No. 1, 1978, pp. 48-57. doi:10.1115/1.3446326

[2] E. Rivin, "Vibration Isolation of Precision Machinery," SV, Sound and Vibration, Vol. 13, No. 8, 1979, pp. 18-23.

[3] A. B Barnes, "Improvement in Machinery for Woven Wire Mesh," Wire Industry, Vol. 51, No. 605, 1984, pp. 403-404.

[4] A. Okayasu, T. Ohta, et al., "Vibration Problems in the LE-7 Liquid Hydrogen Turbo Pump," Proceedings of the $26^{\text {th }}$ AIAA Joint Propulsion Conference, Orlando, Florida, 1990, pp. 1-5.

[5] X. Wang and Z. Zhu, "Ring-Like Metal Rubber Damper," Journal of Aerospace Power, Vol. 12, No. 2, 1997, pp. 143-145.

[6] E. M. Al-Khateeb and J. M. Vance, "Experimental Evaluation of a Metal Mesh Bearing Damper in Parallel with a Structural Support," ASME Paper 2001-GT-0247, Presented at the ASME Turbo Expo, New Orleans, 4-7 June 2001.

[7] M. Zarzour and J. M. Vance, "Experimental Evaluation of a Metal Mesh Bearing Damper," Journal of Engineering for Gas Turbines and Power, Transactions of the ASME, Vol. 122, No. 2, 2000, pp. 326-329.

[8] B. Ertas, E. M. Al-Khateeb and M. John, "Cryogenic Temperature Effects on Metal Mesh Dampers and Liquid Hydrogen Turbo Pump Rotor Dynamics," AIAA Journal \& Proceedings, 2002, AIAA Paper AIAA-2002-4164. 
[9] H. Zuo, Y. H. Chen, H. B. Bai and H. Sun, "The Compression Deformation Mechanism of a Metallic Rubber," International Journal of Mechanics and Materials in De- sign, Vol. 2, No. 3-4, 2005, pp. 269-277. doi:10.1007/s10999-006-9007-x 